\title{
Menger's Paths with Minimum Mergings
}

\author{
Guangyue Han \\ Department of Mathematics \\ University of Hong Kong \\ Pokfulam Road, Hong Kong \\ e-mail: ghan@maths.hku.hk
}

February 25, 2019

\begin{abstract}
Consider an acyclic directed graph with one source and multiple sinks. We prove that one can choose the Merger's paths between the source and the sinks such that the number of mergings between these paths is upper bounded by a constant depending only on the min-cuts between the source and the sinks, regardless of the size and topology of the graph. This result is then applied to the computation and estimation of the minimum number of coding operations required in an acyclic directed communication network.
\end{abstract}

\section{Introductions}

Throughout this paper, we consider an acyclic directed graph $G(V, E)$, where $V$ denotes the set of all the vertices in $G$ and $E$ denotes the set of all the edges in $G$. Using these notations, the edge-connectivity version of Menger's theorem [4] states:

Theorem 1.1 (Menger, 1927). For any $u, v \in V$, the maximum number of pairwise edgedisjoint directed paths from $u$ to $v$ in $G$ equals the min-cut between $u$ and $v$, namely the minimum number of edges in $E$ whose deletion destroys all directed paths from $u$ to $v$.

We call any set consisting of the maximum number of pairwise edge-disjoint directed paths from $u$ to $v$ a set of Menger's paths from $u$ and $v$. Apparently, for fixed $u, v \in V$, there may exist multiple sets of Menger's paths.

For $m$ paths $\beta_{1}, \beta_{2}, \cdots, \beta_{m}$ in $G(V, E)$, we say these paths merge at $e \in E$ if

1. $e \in \cap_{i=1}^{m} \beta_{i}$,

2. there are at least two distinct $f, g \in E$ such that $f, g$ are immediately ahead of $e$ on some $\beta_{i}, \beta_{j}$, respectively. 
We call $e$ together with subsequent shared edges (by all $\left.\beta_{i}\right)$ merged subpath by $\beta_{i}(i=$ $1,2, \cdots, m)$ at $e$. Note that in this paper we will count number of mergings without multiplicities: all the mergings at the same edge $e$ will be counted as one merging at $e$.

For any acyclic directed graph $G(E, V)$ with one source $S \in V$ and $n$ sinks, $R_{1}, R_{2}, \cdots, R_{n}$, whose min-cuts with the source are $c_{1}, c_{2}, \cdots, c_{n}$, respectively. It follows from Menger's theorem that there are $c_{i}$ pairwise edge-disjoint paths (Menger's paths) between $S$ and $R_{i}$. Let $M\left(G, c_{1}, c_{2}, \cdots, c_{n}\right)$ denote the minimum mergings over all the Menger's paths connecting $S$ and $R_{1}, R_{2}, \cdots, R_{n}$. Let $\mathcal{M}\left(c_{1}, c_{2}, \cdots, c_{n}\right)$ denote the largest $M\left(G, c_{1}, c_{2}, \cdots, c_{n}\right)$ over all choices of $G$. We shall prove that

Theorem 1.2. For any $c_{1}, c_{2}, \cdots, c_{n}$,

$$
\mathcal{M}\left(c_{1}, c_{2}, \cdots, c_{n}\right)<\infty
$$

\section{Minimum Mergings}

In the following $G(V, E)$ will be represented through a regular geometric form in the 2 dimensional $x y$ plane (where $x$-axis pointing to the right and $y$-axix pointing downward): all edges in $G$ are represented by straight line segments going downward, i.e., for any edge the $y$-coordinate of the ending point is strictly larger than that of the starting point. Apparently the existence of a regular geometric form of $G(V, E)$ follows from acyclicity of $G$. With the regular gometric form of $G, P_{v}(\cdot)$ will be used to denote the projection to the $y$-axis.

For two sets of pairwise edge-disjoint paths

$$
\alpha=\left\{\alpha_{1}, \alpha_{2}, \cdots, \alpha_{m}\right\}, \quad \beta=\left\{\beta_{1}, \beta_{2}, \cdots, \beta_{n}\right\}
$$

a subpath of $\alpha_{i}$ is called a $\alpha_{i}$-blocking on $\beta$ if this subpath merges with every path in $\beta$. A $\alpha_{i^{-}}$ blocking is called a minimal $\alpha_{i}$-blocking if none of its proper subpaths merges with all paths from $\beta$. Let $a(\cdot), b(\cdot)$ denote the starting point and the ending point of a path, respectively, and let $(\cdot)[s, t]$ denote the subpath of a given path with starting point $s$ and ending point $t$ (here $s$ can take $-\infty$ which represents the starting point of the given path, similarly $t$ can take $\infty$, which represents the ending point of the given path). For two merged subpaths $u, v$, we say $v$ is quasi-reachable by $u$ if there is a sequence of merged subpaths $\gamma_{0}, \gamma_{1}, \cdots, \gamma_{n}$ such that

1. $\gamma_{0}=u, \gamma_{n}=v$

2. For each feasible $k, b\left(\gamma_{2 k+1}\right)$ is ahead of $a\left(\gamma_{2 k}\right)$ on some $\beta_{t(k)}(t(k)$ is some function on $k)$, and $\beta_{t(k)}\left[b\left(\gamma_{2 k+1}\right), a\left(\gamma_{2 k}\right)\right]$ doesn't merge with any paths from $\alpha$;

3. For each feasible $k, b\left(\gamma_{2 k+1}\right)$ is ahead of $a\left(\gamma_{2 k+2}\right)$ on some $\alpha_{h(k)}(h(k)$ is some function on $k$ ).

We say $v$ is reachable by $u$ if other than the three conditions above, we further require that all $h(k)$ 's (see condition 3) are distinct from each other. If $n$ is an even number, we say $v$ is reachable by $u$ from above; if $n$ is an odd number, we say $v$ is reachable by $u$ from below.

With the setting specified above, the following proposition is apparent: 


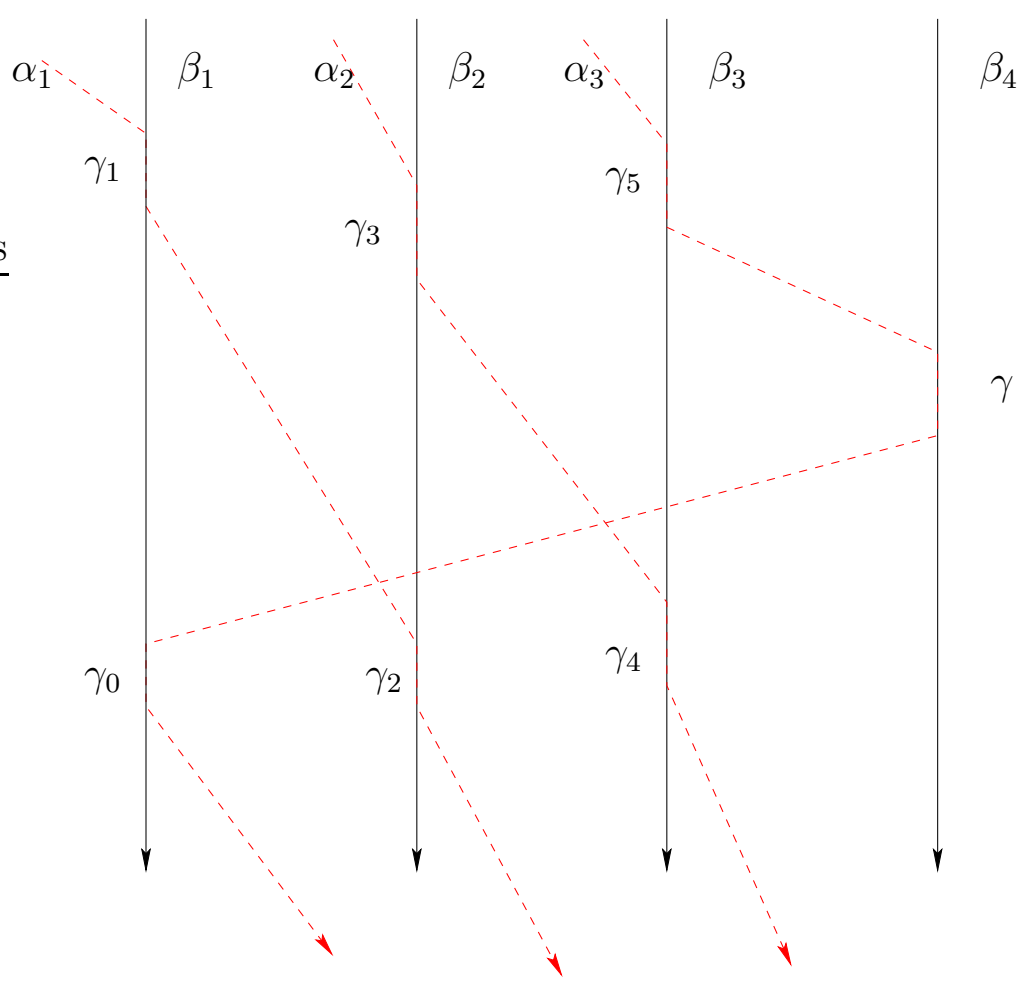

Figure 1: $\alpha$ and $\beta$

Proposition 2.1. If a merged subpath $u$ is reachable by itself from above via a sequence of merged subpaths $\gamma_{0}, \gamma_{1}, \cdots, \gamma_{2 m}=\gamma_{0}$, then one can reroute $\alpha$ to obtain a set of $m$ pairwise edge-disjoint paths, by replacing $\alpha_{h(k)}\left[b\left(\gamma_{2 k+1}\right), \infty\right]$ by $\beta_{t(k)}\left[b\left(\gamma_{2 k+1}\right), a\left(\gamma_{2 k}\right)\right]$ connected with $\alpha_{h(k-1)}\left[a\left(\gamma_{2 k}\right), \infty\right]$.

We call such replacement in Proposition 2.1 a rerouting of $\alpha$ through $\beta$.

Proposition 2.2. Consider a merged subpath $v$ quasi-reachable by a merged subpath $u$ via a sequence of merged subpaths $\gamma_{0}, \gamma_{1}, \cdots, \gamma_{n}$. If none of $\gamma_{i}$ 's is reachable by itself from above, then $v$ is reachable by $u$.

Sketch of the proof. For any $k<l$ such that $h(k)=h(l)$ and $h(k+1), h(k+2), \cdots, h(l-1)$ are all distinct from each other, $v$ is quasi-reachable via a shorter seqence

$$
\gamma_{0}, \cdots, \gamma_{2 k+1}, \gamma_{2 l+2}, \cdots, \gamma_{n}
$$

Continue to find shorter immediate sequences in the similar fashion until all $h(k)$ 's (in condition 3) are all distinct.

It immediately follows from Proposition 2.2 that for three merged subpaths $u, v, w$, if $v$ is reachable from $u$ via $\gamma_{0}, \gamma_{1}, \cdots, \gamma_{n}, w$ is reachable by $v$ via $\tau_{0}, \tau_{1}, \cdots, \tau_{m}$, and none of $\gamma_{i}$ 's and $\tau_{j}$ 's is reachable from itself, then $w$ is also reachable from $u$.

Example 2.3. In Figure [1, $\gamma$ and $\gamma_{i}(i=0,1, \cdots, 5)$ are merged subpaths from $\alpha=$ $\left\{\alpha_{1}, \alpha_{2}, \alpha_{3}\right\}$ and $\beta=\left\{\beta_{1}, \beta_{2}, \beta_{3}, \beta_{4}\right\}$. By definitions, we have 


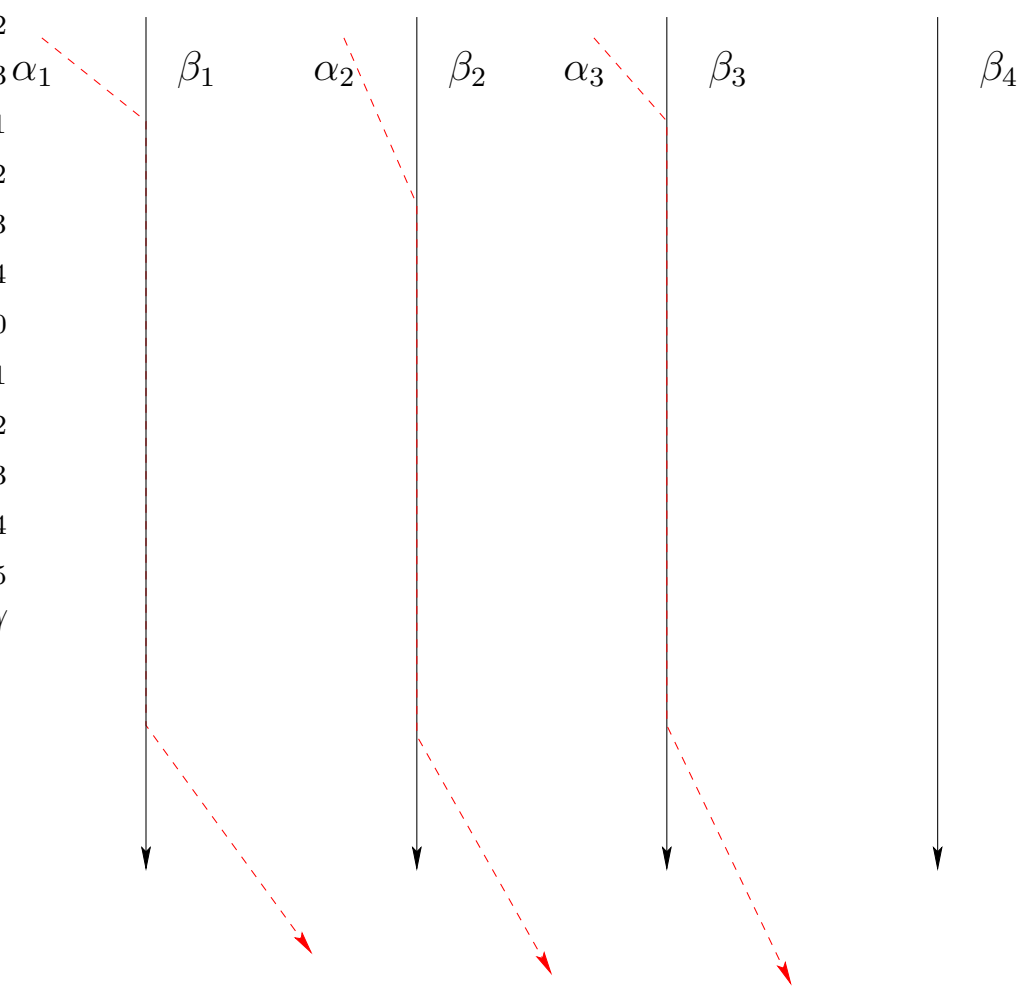

Figure 2: rerouted $\alpha$ through $\beta$

1. $\gamma_{1}, \gamma_{3}, \gamma_{5}$ are reachable from below by $\gamma_{0}$,

2. $\gamma_{3}, \gamma_{5}$ are reachable from below by $\gamma_{2}$,

3. $\gamma_{2}, \gamma_{4}$ are reachable from above by $\gamma_{0}$,

4. $\gamma$ is reachable from above by $\gamma_{0}, \gamma_{2}, \gamma_{4}$.

5. $\gamma_{0}$ is reachable from above by itself, so are $\gamma_{2}, \gamma_{4}$, thus a rerouting of $\alpha$ through $\beta$ is possible by Proposition 2.1 (as shown in Figure 2).

Now let $\alpha_{1}$ be a path and $\beta=\left\{\beta_{1}, \beta_{2}, \cdots, \beta_{n}\right\}$ be a set of $n$ pairwise edge-disjoint paths in $G(V, E)$, respectively. Suppose that

1. $\alpha_{1}, \beta$ take a regular geometric form,

2. for any $j, a\left(\alpha_{1}\right) \notin \beta_{j}, a\left(\beta_{j}\right) \notin \alpha_{1}$.

Remark 2.4. The constraint posed on the starting points by condition 2 allow us to avoid certain pathological situations at the "boundary". For instance, if $a\left(\alpha_{1}\right) \in \beta_{1}$ and the first edge $e_{1}$ of $\alpha_{1}$ is also shared by $\beta_{1}$, then $e_{1}$ will "behave" like a merged subpath, however it is not by the definition of merged subpath. A quick remedy for such case violating condition 2 is to add an "imaginary" edge $e_{0}$ such that $b\left(e_{0}\right)=a\left(e_{1}\right)$ and $e_{0}$ does not intersect with any other edges except at $\left.b\left(e_{0}\right)\right)=a\left(e_{1}\right)$ to form a new $\alpha_{1}$. This way, the starting point of 
this new $\alpha_{1}$ does not violate condition 2. Later in this paper (e.g., before Lemma 2.6), we will have similar constaints posed on the starting points. As illustrated above, any violation of these constaints can always be circumvented by adding certain imaginary edges. We will assume adopting such imaginary edges whenever condition 2 is violated.

Lemma 2.5. For any given $N$, if $\alpha_{1}$ merges with $\beta$ enough times, then there exists $m$, $m \leq n$, and $y_{1}, y_{2} \in \mathbb{R}$, such that $\alpha_{1}$ only merges with $m$ paths from $\beta\left[y_{1}, y_{2}\right]$ (here $\beta\left[y_{1}, y_{2}\right]$ denotes the part of $\beta$ below $y=y_{1}$ and above $y=y_{2}$ ), and there are more than $N$ minimum $\alpha_{1}$-blockings on these $m$ paths.

Proof. We proceed using the induction on $n$.

When $n=1$, the lemma is trivially true.

Now suppose the lemma holds for $n \leq k$. We shall prove that it is also true for $n=k+1$. If $\alpha_{1}$ only merges with $m(m<k+1)$ paths from $\beta$, the lemma immediately follows from the induction assumption. So we can assume $\alpha_{1}$ indeed merges with all $k+1$ paths from beta with merged subpaths $\gamma_{1}, \gamma_{2}, \cdots, \gamma_{l}$ along the direction of $\alpha_{1}$. Let $i_{1}$ be the smallest index such that $\alpha\left[a\left(\gamma_{1}\right), b\left(\gamma_{i_{1}}\right)\right]$ merges with all $k+1$ paths, and similarly $i_{t+1}$ be the smallest index such that $\alpha\left[a\left(\gamma_{i_{t}+1}\right), b\left(\gamma_{i_{t+1}}\right)\right]$ merges with all $k+1$ paths, for $t=1,2, \cdots$. Suppose that we have $M$ such indices. If $M \geq N$, the lemma is automatically true with $m=k+1$. Otherwise, consider subpaths of $\alpha_{1}: \alpha_{1}\left[a\left(\gamma_{1}\right), b\left(\gamma_{i_{1}-1}\right)\right], \alpha_{1}\left[a\left(\gamma_{i_{t}+1}\right), b\left(\gamma_{i_{t+1}-1}\right)\right]$ for $t=1,2, \cdots, M-1$, and $\alpha_{1}\left[a\left(\gamma_{i_{M}+1}\right), b\left(\gamma_{l}\right)\right]$. If $\alpha_{1}$ merges with $\beta$ enough times, then one of abovementioned $\alpha_{1}$ 's subpaths will merge with $k$ paths from $\beta$ enough times. By induction assumption lemma is true as well for this case.

For any $m \leq n$, let $S_{1}, S_{2}, T_{1}, T_{2}$ be subsets of $V$ such that

1. $S_{1}, S_{2}, T_{1}, T_{2}$ consist of $m, m, n, n(m \leq n)$ vertices, respectively,

2. $S_{1} \cap T_{1}=\emptyset$.

Let $M^{*}\left(G, S_{1}, S_{2}, T_{1}, T_{2}, m, n\right)$ denote the minimum number of mergings by all choices of $\alpha$ and $\beta$, where $\alpha=\left\{\alpha_{1}, \cdots, \alpha_{m}\right\}$ is any set of $m$ pairwise edge-disjoint paths, $\beta=\left\{\beta_{1}, \cdots, \beta_{n}\right\}$ be a set of $n$ pairwise edge-disjoint paths in $G(V, E)$, respectively, such that

1. the starting and ending point set of $\alpha$ are $S_{1}, S_{2}$, respectively,

2. the starting and ending point set of $\beta$ are $T_{1}, T_{2}$, respectively,

3. for all $i, j, a\left(\alpha_{i}\right) \notin \beta_{j}, a\left(\beta_{i}\right) \notin \alpha_{j}$.

For any $m \leq n$, let $\mathcal{M}^{*}(m, n)$ denote the largest $M^{*}\left(G, S_{1}, S_{2}, T_{1}, T_{2}, m, n\right)$ over all possible choices of $G, S_{1}, S_{2}, T_{1}, T_{2}$. We then have the following lemma:

Lemma 2.6. For any $m \leq n$,

$$
\mathcal{M}^{*}(m, n)<\infty
$$


Proof. We shall prove that for any such $\alpha$ and $\beta$ in any $G$, there exist $\alpha^{\prime}$, a set of $m$ pairwise edge-disjoint paths, and $\beta^{\prime}$, a set of $n$ pairwise edge-disjoint paths, such that $\alpha^{\prime}, \beta^{\prime}$ have the same set of starting and ending points as $\alpha, \beta$, respectively, and the number of mergings between $\alpha^{\prime}$ and $\beta^{\prime}$ is bounded by a constant which only depends on $m, n$.

Suppose that there are $N$ mergings by $\alpha$ and $\beta$. It suffices to prove that for any $m, n$, if $N$ is larger than a given finite constant (depending only on $m, n$ ), one can always reroute $\alpha, \beta$ to obtain $\alpha^{\prime}$, a set of $m$ pairwise edge-disjoint paths sharing the same set of starting and ending points as $\alpha$, and $\beta^{\prime}$, a set of $m$ pairwise edge-disjoint paths sharing the same set of starting and ending points as $\beta$, such that the number of mergings between $\alpha^{\prime}$ and $\beta^{\prime}$ decreases.

In the following, we assume $\alpha, \beta$ take a regular geometric form.

We proceed by induction. First consider the case when $m=1, n \geq 1$. For this case, if there are $N \geq n+1$ mergings by $\alpha$ and $\beta$, by Pigeonhole principle, there exists $i$ such that $\beta_{i}$ merges with $\alpha_{1}$ at least twice, say at $e$ and $f$. Then we can replace $\alpha_{1}[a(e), a(f)]$, the subpath of $\alpha_{1}$ starting from $a(e)$ to $a(f)$, by $\beta_{i}[a(e), a(f)]$, the subpath of $\beta_{i}$ starting from $a(e)$ to $a(f)$. Let $\alpha_{1}^{\prime}$ be the rerouted $\alpha_{1}$ and $\beta^{\prime}$ be the original $\beta$. Apparently $\alpha_{1}^{\prime}$, the rerouted $\alpha_{1}$, has fewer mergings with $\beta^{\prime}$. It then follows that $\mathcal{M}^{*}(1, n) \leq n+1$ (In fact one can show the equality holds).

Suppose $\mathcal{M}^{*}(m, n)<\infty$ for any $m \leq k, n \geq m$, namely for such $m, n$, whenever the number of mergings by $\alpha$ and $\beta$ is larger than $\mathcal{M}^{*}(m, n)$, rerouting of $\alpha$ or $\beta$ can be done to reduce the number of mergings. We now continue to prove that the lemma is also true for $m=k+1, n \geq m$.

Note that for each $i$, the number of merged (with $\beta$ ) subpaths of $\alpha_{i}$ can be assumed to be "large" given that $M$ is "large". Otherwise, say for instance, $\alpha_{1}$ only has "a few" merged (with $\beta$ ) subpaths, $\gamma_{1}, \gamma_{2}, \cdots, \gamma_{l}$, along $\alpha_{1}$. Then by Pigeonhole principle, at least one of the following:

$$
\beta\left[-\infty, a\left(\gamma_{1}\right)\right], \beta\left[a\left(\gamma_{1}\right), a\left(\gamma_{2}\right)\right], \cdots, \beta\left[a\left(\gamma_{n-1}\right), a\left(\gamma_{n}\right)\right], \beta\left[a\left(\gamma_{n}\right), \infty\right]
$$

will have "many" mergings with $\alpha_{2}, \alpha_{3}, \cdots, \alpha_{m}$. By induction, rerouting of the corresponding part of $\alpha$ or $\beta$ can be done to reduce the number of mergings.

By Lemma 2.5, we can assume, without loss of generality, that for some $m_{0}, m_{0} \leq n$, $\alpha_{1}$ only merges with $\beta_{1}, \beta_{2}, \cdots, \beta_{m_{0}}$, and there are "many" minimal $\alpha_{1}$-blockings on these paths. Pick one such minimal $\alpha_{1}$-blocking $\phi_{0}$ in the middle (so that there are "many" $\alpha_{1^{-}}$ blocking above and below $\phi_{0}$ ), and consider all the reachable merged subpaths by $\phi_{0}$. If all $n$ paths from $\beta$ contain one of the reachable merged subpaths, consider the highest such subpaths on each of $\beta_{1}, \beta_{2}, \cdots, \beta_{n}$, say $\psi_{1}, \psi_{2}, \cdots, \psi_{n}$, respectively. We can assume that none of these highest reachable subpaths belongs to $\alpha_{1}$, otherwise any $\alpha_{1}$-merged subpath in $\phi_{0}$ is reachable by itself from above, then by Proposition 2.1, rerouting of $\alpha_{1}$ can be done to reduce the number of mergings. So with this assumption, at least two of such highest merged subpaths, say $\psi_{i}, \psi_{j}$, will belong to the same path from $\alpha$. Assume that $\psi_{i}$ is ahead of $\psi_{j}$ on this path from $\alpha$. If there is a merged subpath $\psi_{j}^{\prime}$ immediately ahead of $\psi_{j}$ on $\beta_{j}$ (meaning no other merged subpaths in between $\psi_{j}^{\prime}$ and $\psi_{j}$ ), then by definition, $\psi_{j}^{\prime}$ will be reachable by $\phi_{0}$ as well, which contradicts the fact that $\psi_{j}$ is the highest reachable (by $\phi_{0}$ ) merged subpath on $\beta_{j}$. As a consequence, at least one of the highest reachable merged subpaths will 
be in fact the highest merged subpath (regardless of reachability by $\phi_{0}$ ) on some path from $\beta$. Without loss of generality, we assume that $\psi_{n}$ is in fact the highest merged subpath on $\beta_{n}$.

By induction, if $\beta\left[-\infty, a\left(\psi_{n}\right)\right]$ has more than $\mathcal{M}^{*}(m, n-1)$ mergings by $\alpha$ and $\beta_{1}, \beta_{2}, \cdots, \beta_{n-1}$, rerouting can be done to reduce the number of mergings. So we can assume that $\beta\left[-\infty, a\left(\psi_{n}\right)\right]$ has less than $\mathcal{M}^{*}(m, n-1)$ mergings. As a consequence of this, $\beta\left[a\left(\psi_{n}\right), a\left(\phi_{0}\right)\right]$ will have "many" mergings. We further assume that $\psi_{n}$ belongs to $\alpha_{j_{0}}$, which, below $\psi_{n}$ and above $\phi_{0}$, subsequently merges with $\beta$ on $\gamma_{1}, \gamma_{2}, \cdots, \gamma_{l}$ (Note that $l$ can be assumed to be "large"). If within $\beta\left[a\left(\psi_{n}\right), b\left(\gamma_{l}\right)\right]$, for any $i$, any merged subpath immediately above $\gamma_{i}$ (on some path from $\alpha$ ) also belongs to $\alpha_{j_{0}}$, then by induction, rerouting of $\alpha_{j_{0}}$ can be done to reduce the number of mergings on $\beta\left[b\left(\psi_{n}\right), b\left(\gamma_{l}\right)\right]$. So we can assume that there exists $j_{1} \neq j_{0}$ such that $\alpha_{j_{1}}$ has a merged subpath right above some $\gamma_{i_{0}}$, and thus this merged subpath is reachable by $\phi_{0}$. Continue the inductive arguments in the similar fahsion, we can further assume that there exists $j_{k+1} \neq j_{0}, j_{1}, \cdots, j_{k}$ such that $\alpha_{j_{k+1}}$ has a merged subpath right above some reachable merged subpath of $\alpha_{j_{0}}, \alpha_{j_{1}}, \cdots, \alpha_{j_{k}}$. Evetually one can show that above $\phi_{0}, \alpha_{1}$ will have a merged subpath reachable by $\phi_{0}$, which, via Proposition 2.1, implies the number of mergings within $\beta\left[a\left(\psi_{n}\right), a\left(\phi_{0}\right)\right]$ can be reduced by appropriate reroutings.

Now assume that the highest merged subpaths reachable by $\phi_{0}$ do not spread out to all $n$ paths from $\beta$, say only $\beta_{1}, \beta_{2}, \cdots, \beta_{n_{0}}$ (here $n_{0}<n$ ) contain such merged subpaths. Then by the similar inductive argument as above, there exists a merged subpath $\gamma$ well above the lowest minimum $\alpha_{1}$-blocking such that below $\gamma$ all merged subpaths are reachable from $\phi_{0}$, implying below $\gamma$ all paths from $\alpha$ do not merge with $\beta_{n_{0}+1}, \cdots, \beta_{n_{1}}$. Then by induction, rerouting can be done to reduce the number of mergings within $\beta[\gamma, \infty]$.

We exhausted all the cases and then established the lemma.

We are now ready for the proof of Theorem 1.2 .

Proof. To prove Theorem 1.2, it suffices to prove that for each $i$, one can choose pairwise edge-disjoint paths $\alpha_{i, m}, m=1,2, \cdots, c_{i}$, connecting $S$ and $R_{i}$, such that the number of mergings between paths from $\left\{\alpha_{i, m}, i=1,2, \cdots, n, m=1,2, \cdots, c_{i}\right\}$, is upper bounded by a constant only depending on $c_{1}, c_{2}, \cdots, c_{n}$. More specifically, we shall prove that when the number of mergings between Menger's paths $\alpha_{1}, \alpha_{2}, \cdots, \alpha_{n}$ is larger than a given constant (depending only on $c_{1}, c_{2}, \cdots, c_{n}$ ), one can always reroute these Menger's paths such that the number of mergings of the new Menger's paths decreases.

Without loss of generality, we assume that $c_{1} \leq c_{2} \leq \cdots \leq c_{n}$, and we proceed using induction.

For the case when $n=2$, choose $c_{1}, c_{2}$ Merger's paths between $S$ and $R_{1}, R_{2}$, respectively, and add appropriate imiginary edges (see Remark 2.4), then the theorem immediately follows from Lemma 2.6.

Now suppose the theorem is true for $n \leq k$ and consider the case when $n=k+1$. For $i=$ $1,2, \cdots, k+1$, choose a set of Menger's path $\alpha_{i}=\left\{\alpha_{i, 1}, \alpha_{i, 2}, \cdots, \alpha_{i, c_{i}}\right\}$ between $S$ and $R_{i}$, and assume $\alpha_{1}, \alpha_{2}, \cdots, \alpha_{k}$ achieve minimum mergings among them. Let $\beta=\left\{\alpha_{1}, \alpha_{2}, \cdots, \alpha_{k}\right\}$. It suffices to prove that enough mergings between $\beta$ and $\alpha_{k+1}$ will lead to reroutings of some $\alpha_{i}$, which will decrease the number of mergings between $\beta$ and $\alpha_{k+1}$. The projection of the 
terminal points of all merged subpaths, $y_{1}, y_{2}, \cdots, y_{l}$, together with $y_{0}=-\infty, y_{l+1}=\infty$, natually segment the $y$-axis and correspondingly $\beta$. Using Pigeonhole principle, we conclude that for all $i$ 's, at least one of $\beta\left[y_{i}, y_{i+1}\right]$ will have enough mergings with $\alpha_{k+1}$.

In the following, we only consider the part of $\beta$ in between $y=y_{i+1}$ and $y=y_{i}$, and implicitly let $\beta=\beta\left[y_{i}, y_{i+1}\right]$. Without loss of generality, we can assume in this part, $\alpha_{i} \neq \alpha_{j}$ for all $i, j$. Now by Pigeonhole principle again, there exits $j_{1}$ such that $\alpha_{j_{1}}$ has enough mergings with $\alpha_{k+1}$. Consider a complete increasing chain of path sets

$$
\alpha_{j_{1}} \subset \alpha_{j_{2}} \subset \cdots \subset \alpha_{j_{l}}
$$

such that for any $w=1,2, \cdots, l-1, \alpha_{j_{w}} \subset \alpha \subset \alpha_{j_{w+1}}$ for some $\alpha \in \beta$ implies $\alpha=\alpha_{j_{w}}$ or $\alpha_{j_{w+1}}$, and $\alpha_{j_{l}} \subset \alpha$ for some $\alpha \in \beta$ implies $\alpha=\alpha_{j_{l}}$. Now if $\alpha_{k+1}$ merges with paths from $\alpha_{j_{l}} \backslash \alpha_{j_{l-1}}$ enough times, rerouting of $\alpha_{k+1}$ or $\alpha_{j_{l}}$ can be done to reduce the number of merging, otherwise using the segmentation (by the starting points and ending points of merged subpaths in $\alpha_{j_{l}} \backslash \alpha_{j_{l-1}}$ ) argument and the Pigeonhole princple, we can assume $\alpha_{k+1}$ does not merge with $\alpha_{j_{l}}$ at all, and $\alpha_{k+1}$ merges with $\alpha_{j_{l-1}}$ enough times. Continue the argument in the similar fashion as before, we conclude that there exists $\alpha_{j_{v}}(1 \leq v \leq l)$ such that reroutings of $\alpha_{k+1}$ or $\alpha_{j_{v}}$ will reduce the number of mergings. We thus establish the theorem.

Remark 2.7. Theorem 1.2 does not hold for cyclic directed graphs. As shown in Figure 3 , the cyclic graph $G$ has one source $S$ and two sinks $R_{1}, R_{2}$. $\beta_{1}, \beta_{2}$ are Menger's paths between $S$ and $R_{1}$, and $\alpha_{1}, \alpha_{2}$ are Menger's paths between $S$ and $R_{2}$. For an arbitrary $n, \alpha_{1}$ merges with $\beta_{2}$ at $\gamma_{1}, \gamma_{2}, \cdots, \gamma_{n-1}, \gamma_{n}$ subsequently from the bottom to the top. One checks that $\alpha$ and $\beta$ has $n$ mergings, and there is no way to reroute $\alpha$ or $\beta$ to decrease the number of mergings. This implies that $\mathcal{M}(2,2)=\infty$ for cyclic directed graphs.

The following two propositions about $\mathcal{M}$ are obvious, thus their proofs are omitted.

Proposition 2.8. $\mathcal{M}$ is a symmetric on its parameters:

$$
\mathcal{M}\left(c_{1}, c_{2}, \cdots, c_{n}\right)=\mathcal{M}\left(c_{\delta(1)}, c_{\delta(2)}, \cdots, c_{\delta(n)}\right)
$$

where $\delta$ is any permuation on the set of $\{1,2, \cdots, n\}$.

Proposition 2.9. For $m \geq n, c_{1} \leq c_{2} \leq \cdots \leq c_{n}$, and $d_{1} \leq d_{2} \cdots \leq d_{m}$, if $c_{i} \leq d_{m-n+i}$ for $i=1,2, \cdots, n$, then

$$
\mathcal{M}\left(c_{1}, c_{2}, \cdots, c_{n}\right) \leq \mathcal{M}\left(d_{1}, d_{2}, \cdots, d_{m}\right)
$$

Proposition 2.10. For $c_{1} \leq c_{2} \leq \cdots \leq c_{n}$, if $c_{1}+c_{2}+\cdots+c_{n-1} \leq c_{n}$, then

$$
\mathcal{M}\left(c_{1}, c_{2}, \cdots, c_{n}\right)=\mathcal{M}\left(c_{1}, c_{2}, \cdots, c_{n-1}, c_{1}+c_{2}+\cdots+c_{n-1}\right) .
$$

Proof. Consider the case when $G$ has one source $S$ and $n \operatorname{sinks} R_{1}, R_{2}, \cdots, R_{n}$. For each $i=1,2, \cdots, n$, pick a set of Menger's paths $\alpha_{i}=\left\{\alpha_{i, 1}, \alpha_{i, 2}, \cdots, \alpha_{i, c_{i}}\right\}$ from $S$ to $R_{i}$. If any path, say $\beta$, from $\alpha_{n}$ does not share subpath starting from $S$ with any other paths, and first merges with another path $\eta$ at merged subpath $\gamma$, then one can reroute $\eta$ by replacing 


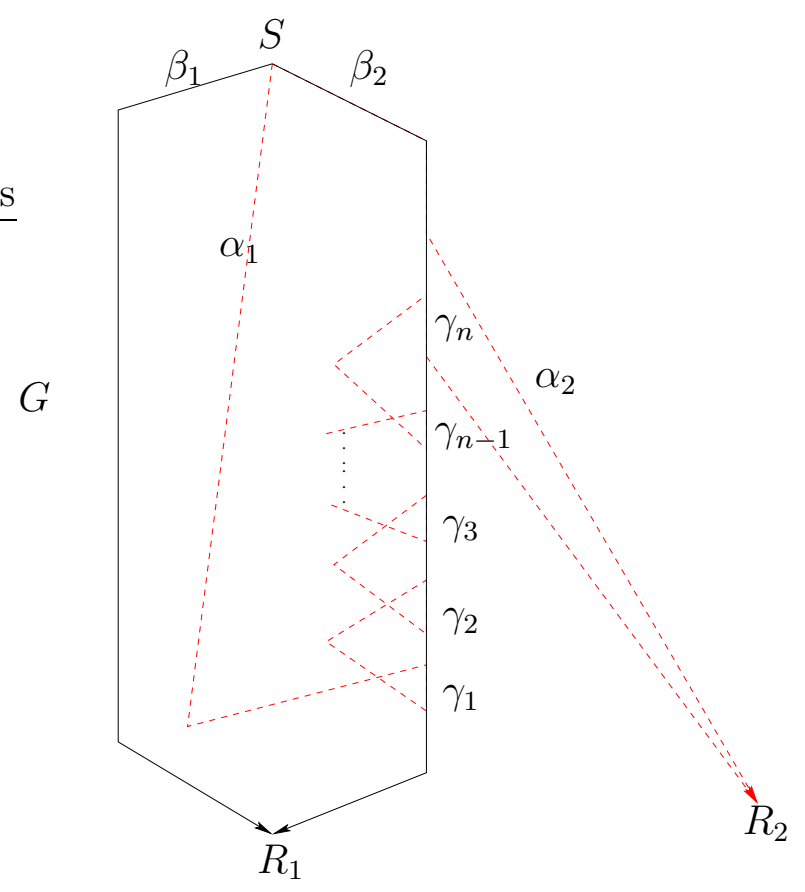

Figure 3: an counterexample

$\eta[S, b(\gamma)]$ by $\beta[S, b(\gamma)]$ to reduce the merging number. Note that such possible reroutings can be done to all the paths from $\alpha_{n}$. As a result of such possible reroutings, at least $c_{n}-\left(c_{1}+c_{2}+\cdots+c_{n-1}\right)$ paths from $\alpha_{n}$ will not merge with any paths from $\alpha_{1}, \alpha_{2}, \cdots, \alpha_{n-1}$. The proposition then immediately follows.

Example 2.11. Suppose that the min-cut between the source $S$ and every sink $R_{i}$ is equal to 1 . For each $i$, pick a Menger's path $\alpha_{i}$ from $S$ to $R_{i}$. Suppose $\alpha_{2}$ last merges with $\alpha_{1}$ at merged subpath $\gamma_{2,1}$ (in this example let "merged subpath" be $S$ if there are no mergings), then one can reroute $\alpha_{2}$ by replacing $\alpha_{2}\left[S, a\left(\gamma_{2,1}\right)\right]$ with $\alpha_{1}\left[S, a\left(\gamma_{2,1}\right)\right]$. Apparently the new $\alpha_{2}$ doesn't merge with $\alpha_{1}$. Suppose we have obtained rerouted $\alpha_{k}$ using alternative subpaths from $\alpha_{1}, \alpha_{2}, \cdots, \alpha_{k-1}$ and there are no mergings between $\alpha_{1}, \alpha_{2}, \cdots, \alpha_{k}$, we now proceed to reroute $\alpha_{k+1}$ to achieve minimum mergings. Suppose $\alpha_{k+1}$ last merges with $\alpha_{1}$ at merged subpath $\gamma_{k+1,1}$, then we reroute $\alpha_{k+1}$ by replacing $\alpha_{k+1}\left[S, a\left(\gamma_{k+1,1}\right)\right]$ with $\alpha_{1}\left[S, a\left(\gamma_{k+1,1}\right)\right]$. Apparently there are no mergings between $\alpha_{1}$ and the newly obtained $\alpha_{k+1}$. For the new $\alpha_{k+1}$, suppose it last merges with $\alpha_{2}$ at the merged subpath $\gamma_{k+1,2}$, we then further reroute it by replacing $\alpha_{k+1}\left[S, a\left(\gamma_{k+1,2}\right)\right]$ with $\alpha_{1}\left[S, a\left(\gamma_{k+1,2}\right)\right]$. One checks that the new $\alpha_{k+1}$ doesn't merge with $\alpha_{1}, \alpha_{2}$. We continue to reroute $\alpha_{k+1}$ using appropriate subpaths of $\alpha_{3}, \alpha_{4}, \cdots, \alpha_{k}$ in a similar fashion, and one checks that the final $\alpha_{k+1}$ we obtain eventually doesn't merge with $\alpha_{1}, \alpha_{2}, \cdots, \alpha_{k}$. Inductively apply the rerouting procedure to all $\alpha_{i}$, we can obtain a new set of paths: $\alpha_{1}, \alpha_{2}, \cdots, \alpha_{n}$, which don't merge among themselves and this shows that $\mathcal{M}(1,1, \cdots, 1)=0$.

Example 2.12. Consider the case when we have two sinks $R_{1}, R_{2}$ only and the min-cut between the source $S$ and every sink is equal to 2. For $i=1,2$, pick a set of Menger's paths $\alpha_{i}=\left\{\alpha_{i, 1}, \alpha_{i, 2}\right\}$ from $S$ to $R_{i}$. We shall go through certain rerouting procedure to reduce the number of mergings among these 4 paths. 
For $i=1,2$, suppose $\alpha_{2, i}$ merges with $\alpha_{1}$ at $\gamma_{2, i, 1}, \gamma_{2, i, 2}, \cdots, \gamma_{2, i, l_{i}}$ (again, in this example, any "merged subpath" means $S$ if, in fact, it is not a valid merged subpath). If two adjacent merged subpaths $\gamma_{2, i, j}, \gamma_{2, i, j+1}$ are subpaths of the same path, say $\alpha_{1,1}$, from $\alpha_{1}$, we can reroute $\alpha_{1,1}$ by replacing $\alpha_{1,1}\left[a\left(\gamma_{2, i, j}\right), b\left(\gamma_{2, i, j+1}\right)\right]$ by $\alpha_{2, i}\left[a\left(\gamma_{2, i, j}\right), b\left(\gamma_{2, i, j+1}\right)\right]$. Apparently the resulted new $\alpha_{1,1}$ has fewer mergings with $\alpha_{2}$. With such possible reroutings, we can assume that for $i=1,2, \alpha_{2, i}$ merges with $\alpha_{1,1}, \alpha_{1,2}$ alternately.

If $\gamma_{2,1, l_{1}}, \gamma_{2,2, l_{2}}$ belong to different paths from $\alpha_{1}$, without loss of generality, say $\alpha_{1,1}, \alpha_{1,2}$, respectively, reroute $\alpha_{2,1}$ by replacing $\alpha_{2,1}\left[S, b\left(\gamma_{2,1, l_{1}}\right)\right]$ with $\alpha_{1,1}\left[S, b\left(\gamma_{2,1, l_{1}}\right)\right]$, and reroute $\alpha_{2,2}$ by replacing $\alpha_{2,2}\left[S, b\left(\gamma_{2,2, l_{2}}\right)\right]$ with $\alpha_{1,2}\left[S, b\left(\gamma_{2,2, l_{2}}\right)\right]$. For this case, the rerouted $\alpha_{2}$ doesn't merge with $\alpha_{1}$.

Now consider the case when $\gamma_{2,1, l_{1}}, \gamma_{2,2, l_{2}}$ belong to the same path from $\alpha_{1}$, say $\alpha_{1,1}$. Without loss of generality, assume $\gamma_{2,1, l_{1}}$ is ahead of $\gamma_{2,2, l_{2}}$ on $\alpha_{1,1}$. Then $\gamma_{2,2, l_{2}-1}$ will be on $\alpha_{1,2}$. So we can reroute $\alpha_{2,1}$ by replacing $\alpha_{2,1}\left[S, b\left(\gamma_{2,1, l_{1}}\right)\right]$ by $\alpha_{1,1}\left[S, b\left(\gamma_{2,1, l_{1}}\right)\right]$, and reroute $\alpha_{2,2}$ by replacing $\alpha_{2,2}\left[S, b\left(\gamma_{2,2, l_{2}-1}\right)\right]$ by $\alpha_{1,2}\left[S, b\left(\gamma_{2,2, l_{2}-1}\right)\right]$. In this case, the rerouted $\alpha_{2}$ merges with $\alpha_{1}$ once at $\gamma_{2,2, l_{2}}$.

The analysis for the two cases above shows that $\mathcal{M}(2,2)=1$, and further together with Proposition 2.10, it also shows $\mathcal{M}(2, m)=1$ for $m \geq 2$.

Example 2.13. Consider the case when $G$ has one source $S$ and two sinks $R_{1}, R_{2}$, and the min-cut between the source $S$ and every sink is equal to 3 . For each sink $R_{i}$, pick a set of Menger's paths $\alpha_{i}=\left\{\alpha_{i, 1}, \alpha_{i, 2}, \alpha_{i, 3}\right\}$. By Proposition [2.9, we can assume every path from $\alpha_{1}$ merges with certain paths from $\alpha_{2}$ and vice versa. As shown in the proof of Proposition 2.10, we can further assume $\alpha_{1, i}$ shares subpath starting from $S$ with $\alpha_{2, i}$ for $i=1,2,3$ after possible reroutings. Now if every path from $\alpha_{1}$, after such reroutings, merges with some path from $\alpha_{2}$, for instance, $\alpha_{1, i}$ first merges with $\alpha_{2, \delta(i)}$ at merged subpath $\gamma_{i}$, here $\delta$ denotes certain mapping from $\{1,2,3\}$ to $\{1,2,3\}$, then there exists $m(m \leq 3)$ such that $\delta^{m}(1)=1$. We further choose $m$ to be the smallest such "period". In this case rerouting of $\alpha_{2}$ can be done by replacing $\alpha_{2, \delta^{j}(1)}\left[S, b\left(\gamma_{\delta^{j-1}(1)}\right)\right]$ by $\alpha_{1, \delta^{j-1}(1)}\left[S, b\left(\gamma_{\delta^{j-1}(1)}\right)\right]$ for $j=1, \cdots, m$ to reduce the merging number. So, without loss of generality, we can assume, after further possible reroutings, $\alpha_{1,3}$ does not merge with any other paths, and $\alpha_{2,1}$ doesn't merge with any other paths either by similar argument. Now paths $\alpha_{1,1}, \alpha_{1,2}$ will only merge with paths $\alpha_{2,2}, \alpha_{2,3}$. As argued in Example 2.12, path $\alpha_{1, j}(j=1,2)$ can be assumed to merge with paths $\alpha_{2,2}, \alpha_{2,3}$ alternately, and similarly path $\alpha_{2, j}(j=2,3)$ can be also assumed to merge with paths $\alpha_{1,1}, \alpha_{1,2}$ alternately. Exhausting all the possible patterns, we conclude that $\mathcal{M}(3,3)=5$, and the minimum merging number is achieved by the Figure 4 .

\section{Applications in Network Coding}

In this section, we follow the framework of Chapter 2 (on single-source-multiple-sinks network) in [6].

By a communication network, we refer to an acyclic directed graph $G(E, V)$ (allowing multiple directed edges between two nodes) with one source $S$ and multiple sinks $R_{1}, R_{2}, \cdots, R_{n}$. A directed edge is called a channel in the communication network $G$, which represents a noiseless communication link on which one unit of information can be transmitted per unit time. 


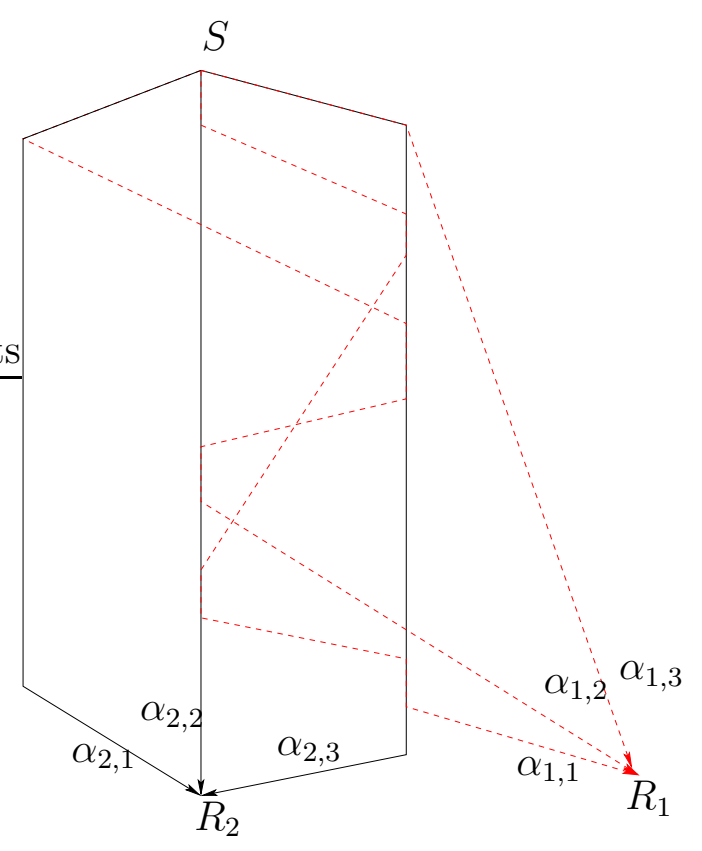

Figure 4: an example achieving minimum mergings

The multiplicity of the channels from a node $i$ to another node $j$ represents the capacity of direct transmission from $i$ to $j$. In other words, every single channel has capacity 1 .

An information unit is represented by an element of a certain finite field $F$ (also called base field). A finite amount of information is generated at the source $S$ and multicast to the sinks $R_{1}, R_{2}, \cdots, R_{n}$ on the network in the multihop fashion where every node can pass on any of its received data to other nodes along the channels. More specifically, the source node S generates a message $\mathbf{x} \in F^{\omega}$ (therefore consisting of $w$ information bits) and sends it out by transmitting a symbol in $F$ over every outgoing channel. Message propagation through the network is achieved by the transmission of a symbol $f_{e}(x) \in F$ over every channel $e$ in the network. An intermediate node (other than the source and the sinks) does not necessarily receive complete information to identify the value of the whole message $\mathbf{x}$. It simply maps the received symbols from all the incoming channels to a symbol for each outgoing channel. A network code is specified by such an encoding mechanism for every channel.

We aim to recover the complete generated information generated at each sink $R_{i}$. We are naturally interested in information transmission rate from the source to each sink, which measures how fast each sink node can receive the complete information. A natural bound on the information transmission rate from $S$ to $R_{i}$ is the min-cut (also called max-flow bound) between them. The advantage of network coding over the traditional routing mechanism was first demonstrated together the fundamental concept of network coding was first introduced for satellite communication networks in [5] and then fully developed in [1], where in the latter the term "network coding" was coined. In 2000, Li, et. al. [3] introduced linear network coding and showed that for large enough base field $F$, such coding scheme suffices to achieve the max-flow bound for each sink simultaneously (their coding scheme works for a more general setting).

Further efforts on linear multicast [2] showed that as long as $|F| \geq n$, the number of sinks, 
the max-flow bound on information transmission rate for each sink can always be achieved. The rough idea of the construction algorithm in [2] can be rephrased as follows. Instead of working on the whole network $G$, we choose a set of Menger's paths from the source to every sink and construct coding scheme on all these Menger's paths. Note that appropriate linear coding operations are only required on merged subpaths among these Menger's paths (for other paths, the traditional store-and-forward routing is enough), and such coding scheme can be proven to exist as long as $|F| \geq n$. It then immediately follows from Theorem 1.2 that the minimum number of coding operations needed to achieve the max-flow bound for all the sinks under linear network coding setting is finite.

Theorem 3.1. For any acyclic directed network $G$ with one source and multiple sinks, where each of the sinks has fixed min-cut with the source, there exists a sub-network $G^{\prime}$ such that only finite number of linear network coding operations on $G^{\prime}$ can guarantee that all the sinks receive the complete information generated at the source, regardless of the size or topology of $G$.

\section{References}

[1] R. Ahlswede, N. Cai, S.-Y. R. Li, and R. W. Yeung. Network Information Flow. IEEE Trans. Inform. Theory, vol. IT-46, pp. 1204-1216, 2000.

[2] S. Jaggi, P. Sanders, P.A. Chou, M. Effros, S. Egner, K. Jain, and L.M.G.M. Tolhuizen. Polynomial time algorithms for multicast network code construction IEEE Trans. Inform. Theory, vol. IT-51, June 2005, pp. 1973 - 1982.

[3] S.-Y. R. Li, R. W. Yeung, and N. Cai. Linear network coding. IEEE Trans. Inform. Theory, vol. IT-49, pp. 371C381, 2003.

[4] K. Menger. Zur allgemeinen Kurventhoerie. Fund. Math., 10:96-115.

[5] R. W. Yeung and Z. Zhang. Distributed source coding for satellite communications. IEEE Trans. Inform. Theory, vol. IT-45, pp. 1111C1120, 1999.

[6] Raymond W. Yeung, Shuo-Yen Robert Li, Ning Cai, Zhen Zhang. Network Coding Theory, now Publishers Inc., 2005. 